\title{
Relationship between genotypes of longevity genes and developmental speed in Drosophila melanogaster
}

\author{
ISAMU YONEMURA, TOMIO MOTOYAMA**, HAYATO HASEKURA \& \\ BARRY BOETTCHER $\dagger$ \\ Department of Legal Medicine, Shinshu University School of Medicine, Matsumoto, 390 Japan, **Department of \\ Physiology, College of Environmental Health, Azabu University, Sagami-hara, 229 Japan and tDepartment of Biological \\ Sciences, The University of Newcastle, New South Wales, 2308 Australia
}

\begin{abstract}
Hatching time (the period between egg-laying and hatching) and emerging time were surveyed and their relationship with the adult life span was investigated. A relationship between emerging time and adult life span was clearly evident: early emergers were often long-lived. This relation is considered to have a genetic basis because all the larvae in a group were bred in the same culture bottle. Thus, the longevity genes involved also appear to have control over the rate of development. No significant relation was observed between hatching time and adult life span or between hatching time and emerging time. These results suggest that the function of the longevity genes begins at the larval or pupal stage before emergence, and that adult life spans differentiate at this time.
\end{abstract}

Keywords: developmental speed, Drosophila melanogaster, life span, longevity genes, longevity potential.

\section{Introduction}

The speed of larval development in Drosophila is known to have a strong relationship with quantitative traits in the aduit: e.g. bristle number, size of the adult, or life span. Wattiaux (1962) observed in a strain bred under uniform conditions, that individuals with a faster rate of development showed a significantly larger number of bristles.

The relationship between size and rate of development may differ according to the experimental conditions. Lints \& Lints (1971) reported that a high larval density delayed growth and diminished imaginal size. Burcombe \& Hollingsworth (1970) bred Drosophila and found that development was slower and size larger when the flies were grown at a lower temperature.

With regard to life span, a slow rate of development is considered to be associated with a long life. The rate of development can be changed largely by controlling temperature, larval density, or nutrition. In general, breeding at lower temperature slows the rate and flies take longer to emerge, but it also lengthens the life span by comparison with flies bred at a higher temperature and with a faster rate of development (Alpatov \& Pearl, 1929; Burcombe \& Hollingsworth, 1970; Lints \&
Lints, 1971; Cohet, 1975). In addition, a higher laval density was observed to lengthen the developmental period and to prolong the adult life span (Lints \& Lints, 1971).

As all of these studies reported that a longer preimaginal period was associated with a longer life span, Lints \& Lints (1971) and Burcombe \& Hollingsworth (1970) considered that changes in the rate of development, due to environmental conditions, influenced the expression of the genes that control the adult life span.

Detailed comparison of their experimental conditions and their results, however, revealed irregular relationships between life span and environmental factors, e.g. temperature and/or nutrition; the stated relations held only in a suitable environmental range and the life span was shortened abruptly near either end of the range (Economos \& Lints, 1984a,b; Lints, 1989). In Tribolium castaneum, early emergers were also reported to be longer-lived (Soliman \& Lints, 1982).

The relationship between life span and rate of development still remains an important and interesting phenomenon to be investigated. We have demonstrated in Drosophila melanogaster that a pair of autosomal genes and a pair of $X$ chromosomal allelic major genes 
control the life span of adult flies. These longevity genes are called the Jm system (Yonemura et al., 1989; Yonemura et al., 1990). Luckinbill et al. (1987) indicated the locations of genes that control the life span of D. melanogaster as chromosomes 1 and 3 . We have now mated two purebred strains of $D$. melanogaster with known $\mathrm{Jm}$ genotypes to produce a generation with a segregation of life spans, in an attempt to clarify the relationships between developmental speed (hatching speed and emerging speed) and Jm longevity genes.

\section{Materials and methods}

Two highly purebred lines of $D$. melanogaster, described in previous reports (Yonemura et al., 1989; Yonemura et al., 1990), were used as the parental lines in this study. The short-lived strain was adopted as $P_{1}$ and the long-lived as $\mathrm{P}_{2} ; \mathrm{P}_{1}$ males and $\mathrm{P}_{2}$ females were mated to produce the $F_{1}$; back-cross mating between $P_{1}$ males and $F_{1}$ females and between $P_{1}$ females and $F_{1}$ males produced the $B_{1-1}$ and $B_{1-3}$ generations. The $B_{1-3}$ generation was surveyed for the relationship between emerging time (period from egg laying to emerging) and adult life span: Emerging speed/Life span Experiment. The $\mathrm{B}_{1-1}$ generation was tested for the relation between hatching time (period from egg laying to hatching) and adult life span: Hatching speed/ Life span Experiment.

In previous reports, the autosomal longevity locus was named $\mathrm{Jm} \mathrm{A}$, the $X$ chromosomal locus $\mathrm{Jm} X$, and the tentative $Y$ chromosomal locus, $\mathrm{Jm} Y$. Thus, the Jm genotype for $\mathrm{P}_{1}$ males is $A 1 A 1 X 1 Y 1$, that for $\mathrm{P}_{1}$ females is $A 1 A 1 X 1 X 1, \mathrm{P}_{2}$ males $A 2 A 2 X 2 Y 2$, and $\mathrm{P}_{2}$ females $A 2 A 2 X 2 X 2$. The $\mathrm{B}_{1-1}$ generation segregates into four genotypes in both sexes, each with a specific life span. $\mathrm{B}_{1-3}$ segregates two genotypes: $A 1 A 1 X 1 Y 1$ and $A 1 A 2 X 1 Y 1$ in males and $A 1 A 1 X 1 X 2$ and $A 1 A 2 X 1 X 2$ in females, those with $\mathrm{Jm} A 2$ are long-lived in both sexes (Yonemura et al., 1989; Yonemura et al., 1990).

\section{Emerging speed/Life span Experiment}

Mass-mating of 30 individuals of $P_{1}$ virgin females and $30 F_{1}$ males was made in each of six culture bottles, each containing $8 \mathrm{ml}$ of food, consisting of 8 per cent dry yeast and 5 per cent sugar. Females were allowed to lay eggs for $8 \mathrm{~h}$ preliminarily. These eggs were discarded to avoid an uneven developmental stage. Eggs to be tested were laid in the next $4 \mathrm{~h}$ and were incubated at $27^{\circ} \mathrm{C}$. Emerging flies were collected every $8 \mathrm{~h}$ (at 8:00, 16:00, and 24:00 hours). Adult flies were divided into sexes under ether anaesthesia, with 30 individuals of each sex being distributed into plastic breeding tubes, and cultured with the above food at $27^{\circ} \mathrm{C}$. The dead were counted and removed every day and food was renewed at the same time. The plastic tube was renewed weekly. Further details of materials and methods and the symbols for the generations may be referred to in our previous reports (Yonemura et al., 1989; Yonemura et al., 1990).

\section{Hatching speed/Life span Experiment}

Thirty $\mathrm{P}_{1}$ males and $30 \mathrm{~F}_{1}$ virgin females were mated in each of six bottles. They were allowed to lay eggs for

Table 1 Emerging time and mean adult life span in $\mathrm{B}_{1-3}$ generation where longevity genotypes ( $\mathrm{Jm} A 1 A 1$ and $\mathrm{Jm} A 1 A 2$ ) segregate. The percentage of the long-lived (presumed $A 1 A 2$ ) individuals (segregation ratio) in each emerging time group is also shown

\begin{tabular}{|c|c|c|c|c|c|c|c|}
\hline \multirow[b]{2}{*}{$\begin{array}{l}\text { Time } \\
\text { group }\end{array}$} & \multirow[b]{2}{*}{$\begin{array}{l}\text { Emerging } \\
\text { time }(\mathbf{h})\end{array}$} & \multicolumn{3}{|c|}{ Male } & \multicolumn{3}{|c|}{ Female } \\
\hline & & No. & $\begin{array}{l}\text { Mean } \pm \text { s.d. } \\
\text { (days) }\end{array}$ & $\begin{array}{l}A 1 A 2 \\
\langle \%\rangle\end{array}$ & No. & $\begin{array}{l}\text { Mean } \pm \text { s.d. } \\
\text { (days) }\end{array}$ & $\begin{array}{l}A l A 2 \\
(\%)\end{array}$ \\
\hline 1 & 184 & 1 & 41.0 & 100 & 33 & $49.3 \pm 8.8$ & 84.8 \\
\hline 2 & 192 & 25 & $39.4 \pm 10.3$ & 76.0 & 113 & $48.2 \pm 9.9$ & 80.5 \\
\hline 3 & 200 & 93 & $37.6 \pm 8.3$ & 79.6 & 134 & $40.6 \pm 11.6$ & 50.7 \\
\hline 4 & 208 & 146 & $30.8 \pm 9.4$ & 50.0 & 93 & $35.0 \pm 11.4$ & 29.0 \\
\hline 5 & 216 & 97 & $24.8 \pm 9.5$ & 25.8 & 26 & $34.5 \pm 13.9$ & 34.6 \\
\hline 6 & 224 & 26 & $23.9 \pm 9.9$ & 23.1 & 10 & $22.9 \pm 14.9$ & 20.0 \\
\hline 7 & 232 & 13 & $22.1 \pm 12.5$ & 23.1 & 2 & $30.5 \pm 12.0$ & 0 \\
\hline 8 & 240 & 7 & $19.1 \pm 9.2$ & 0 & 4 & $38.8 \pm 9.9$ & 25.0 \\
\hline 9 & 248 & 2 & $14.0 \pm 4.2$ & 0 & 1 & 52.0 & 100 \\
\hline Total & & 410 & $30.5 \pm 10.9$ & 49.0 & 416 & $41.4 \pm 12.5$ & 54.6 \\
\hline
\end{tabular}


$8 \mathrm{~h}$ preliminarily, and 40 min experimentally. Larvae were collected every hour after $16.5 \mathrm{~h}$. The numbers of castoff shells were checked microscopically. The larvae were raised in separate bottles to observe the relationship between hatching time and adult life span.

\section{Results}

\section{Emerging speed/Life span Experiment}

Emerging time was taken as the period from the middle of the egg laying period to the middle of the period when emerging flies were collected. Emerging started at $184 \mathrm{~h}$ and lasted for 4 days. The life span was measured for 410 males (average: 30.5 days) and 416 females (average: 41.4 days). Table 1 shows the mean adult life span in every emerging time group. In general, early emergers lived longer than late emergers, however, there were a few long survivors among the lateemerging females.

The distributions of deaths (mortality graphs) in all emerging time groups are presented in Fig. 1 (males) and Fig. 2 (females) (emerging times for groups 7-9 are summed). Graphs for total males and total females are shown at the top of both figures: both graphs give two peaks, with the mean life span falling in between. The left peak corresponds to Jm genotype $A 1 A 1$ and the right peak to $A 1 A 2$. The segregation ratio $A 1 A 1$ : $A 1 A 2=51.0: 49.0$ in males and $A 1 A 1: A 1 A 2=$ 45.4:54.6 in females. The percentages of assumed $A 1 A 2$ individuals in emerging time groups are presented in Table 1.

Regression equations of the life span ( $Y$, day) on emerging time ( $X$, hour), and correlation coefficients, for males $(m)$ and females $(f)$ were calculated as:

$$
\begin{aligned}
& Y_{\mathrm{m}}=142.2-0.53 X_{\mathrm{m}}(r=-0.50, P<0.001), \\
& Y_{\mathrm{f}}=137.2-0.48 X_{\mathrm{f}}(r=-0.40, P<0.001) .
\end{aligned}
$$

The correlation was highly significant in both sexes.

The regression equations for the percentage of $A 1 A 2$ ( $Y$, per cent) on emerging time ( $X$, hour) for males $(\mathrm{m})$ and females ( $\mathrm{f}$, time groups 7-9 being pooled) are:

$$
\begin{aligned}
& Y_{\mathrm{m}}=416.3-1.74 X_{\mathrm{m}}(r=-0.95, P=0.001), \\
& Y_{\mathrm{f}}=289.9-1.16 X_{\mathrm{f}}(r=-0.86, P=0.014) .
\end{aligned}
$$

Significant negative correlations were observed in both sexes. This shows that long-lived individuals, with genotype $A 1 A 2$, emerge earlier than short-lived $A 1 A 1$ individuals. It is an indication that $\mathrm{Jm}$ longevity genes influence developmental speed in the pre-imaginal stage because the relationship was observed within a generation bred in the same culture bottles.

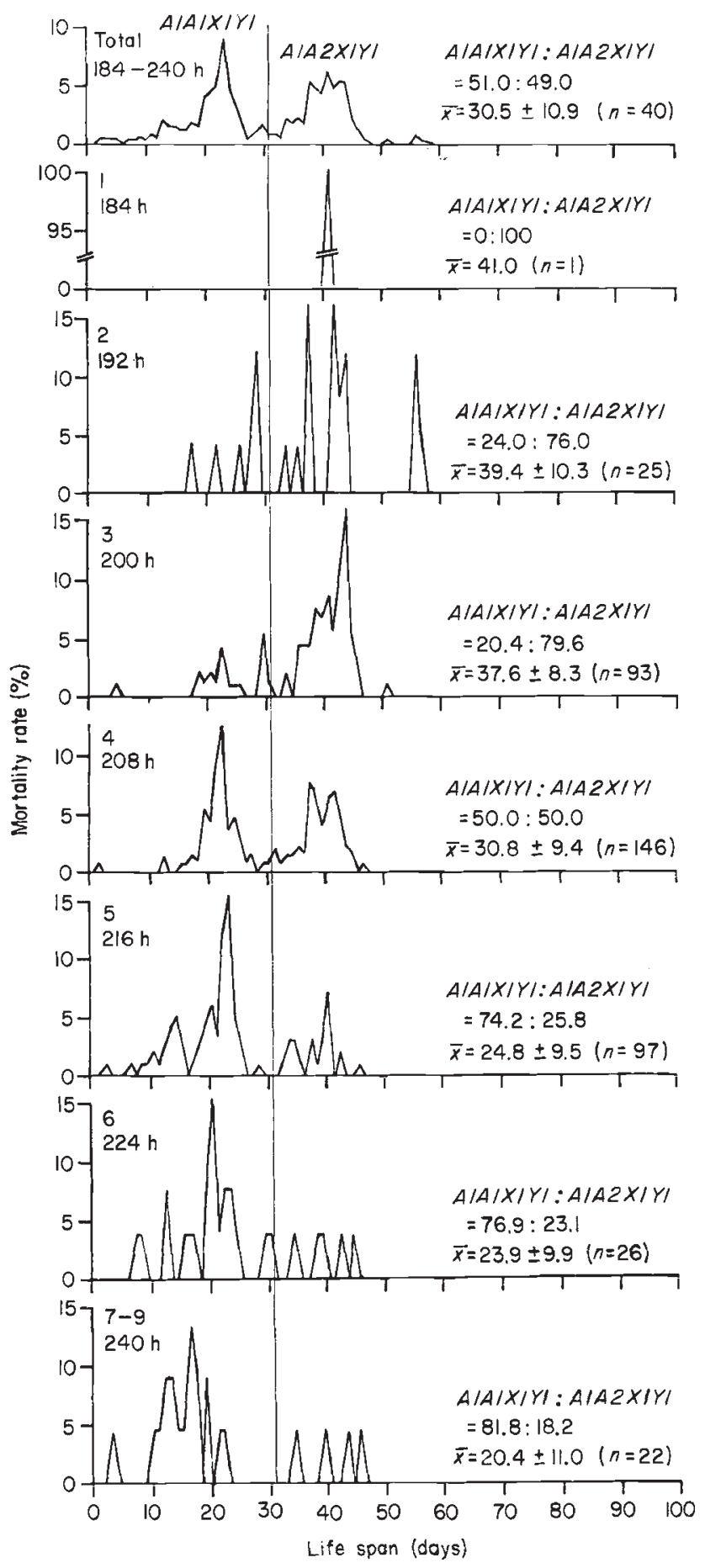

Fig. 1 Mortality graphs (distributions of death rates) for emerging time groups in the $\mathrm{B}_{1-3}$ generation where the shortlived genotype $(A 1 A 1 X 1 Y 1)$ and the long-lived genotype $(A 1 A 2 X 1 Y 1)$ segregate in males. The vertical line in the middle of the graphs indicates the mean life span in the total which divides life span into short and long. See text for the mating procedure to produce the $\mathrm{B}_{1-3}$ generation. 


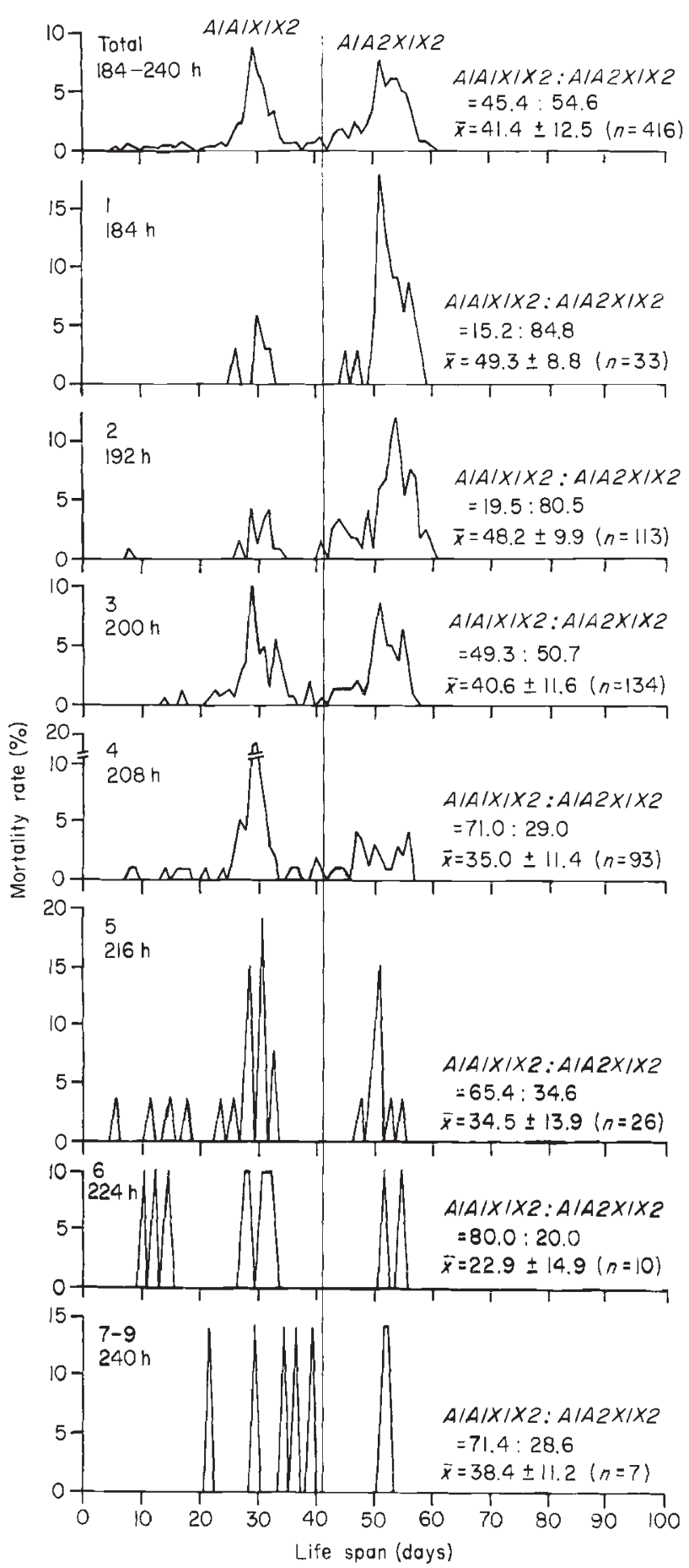

Fig. 2 Mortality graphs (distributions of death rates) for emerging time groups in the $\mathrm{B}_{1-3}$ generation where the shortlived genotype $(A 1 A 1 X 1 X 2)$ and the long-lived genotype $(A 1 A 2 X 1 X 2)$ segregate in females. The vertical line in the middle of the graphs indicate the mean life span in the total which divides life span into short and long. See text for the mating procedure to produce the $B_{1-3}$ generation.
Table 2 shows coefficients of the regression equation $(Y=a+b X)$ of adult life span ( $Y$, day) on emerging time $(X$, hour) in six culture bottles in which identical tests were undertaken in parallel. A significant correlation $(P<0.05)$ was observed in all bottles except bottle number 6 , where a few individuals were unexpectedly long-lived although they were late emergers. In all bottles the mean life span reflected the percentage of the long-lived $(A 1 A 2)$ progeny which segregated (data omitted).

\section{Hatching speed/Life span Experiment}

Hatching took place mainly between 16.5 and $17.5 \mathrm{~h}$ after egg laying and no later than $23.5 \mathrm{~h}$. Table 3 (males) and Table 4 (females) show the hatching time, emerging time, and mean adult life span for each hatching time group.

First, the regression equations of life span ( $Y$, day) on emerging time $(X$, hour) for males $(\mathrm{m})$ and females (f) were calculated as:

$$
\begin{aligned}
& Y_{\mathrm{m}}=135.6-0.56 X_{\mathrm{m}}(r=-0.42, P=0.013), \\
& Y_{\mathrm{f}}=182.2-0.84 X_{\mathrm{f}}(r=-0.35, P=0.056) .
\end{aligned}
$$

In addition, earlier emergers generally lived longer; this tendency was significant in males, but not quite so significant in females (perhaps because of the relatively small numbers involved). Hatching time was grouped into three periods: before $16.5 \mathrm{~h}$ (calculated as $16 \mathrm{~h}$ ), 16.5-17.5 $\mathrm{h}(17 \mathrm{~h})$, and 17.5-23.5 h (18 h).

Secondly, regression equations of life span ( $Y$, day) on hatching time $(X$, hour) for males $(\mathrm{m})$ and females (f) were calculated as:

$$
\begin{aligned}
& Y_{\mathrm{m}}=16.2+0.96 X_{\mathrm{m}}(r=0.04, P=0.805), \\
& Y_{\mathrm{f}}=36.1-0.16 X_{\mathrm{f}}(r=-0.01, P=0.977) .
\end{aligned}
$$

These values indicate that there is no significant correlation between hatching time and adult life span.

Thirdly, regression equations of emerging time ( $Y$, hour) on hatching time $(X$, hour) for males $(\mathrm{m})$ and females (f) were as follows:

$Y_{\mathrm{m}}=131.0+3.00 X_{\mathrm{m}}(r=0.20, P=0.255)$,

$Y_{\mathrm{f}}=179.7-0.12 X_{\mathrm{f}}(r=-0.03, P=0.862)$.

Here again, no significant correlation was observed between hatching time and emerging time. Therefore in the egg stage, the rate of development has been found to bear no relation to longevity genotypes.

\section{Discussion and conclusion}

The inter-relationships between quantitative traits and the rate of development in $D$. melanogaster have been 
Table 2 Coefficients of regression line $(Y=a+b X)$ of adult life span ( $Y$, day) on emerging time $(X$, hour) in six culture bottles

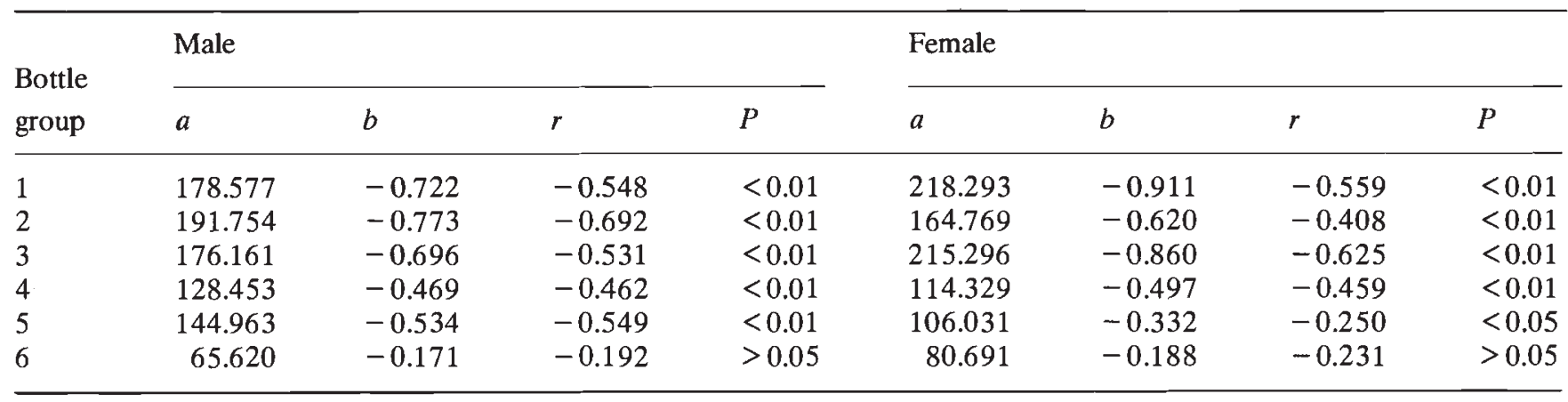

Table 3 The mean adult life span and emerging time for each hatching time group (males)

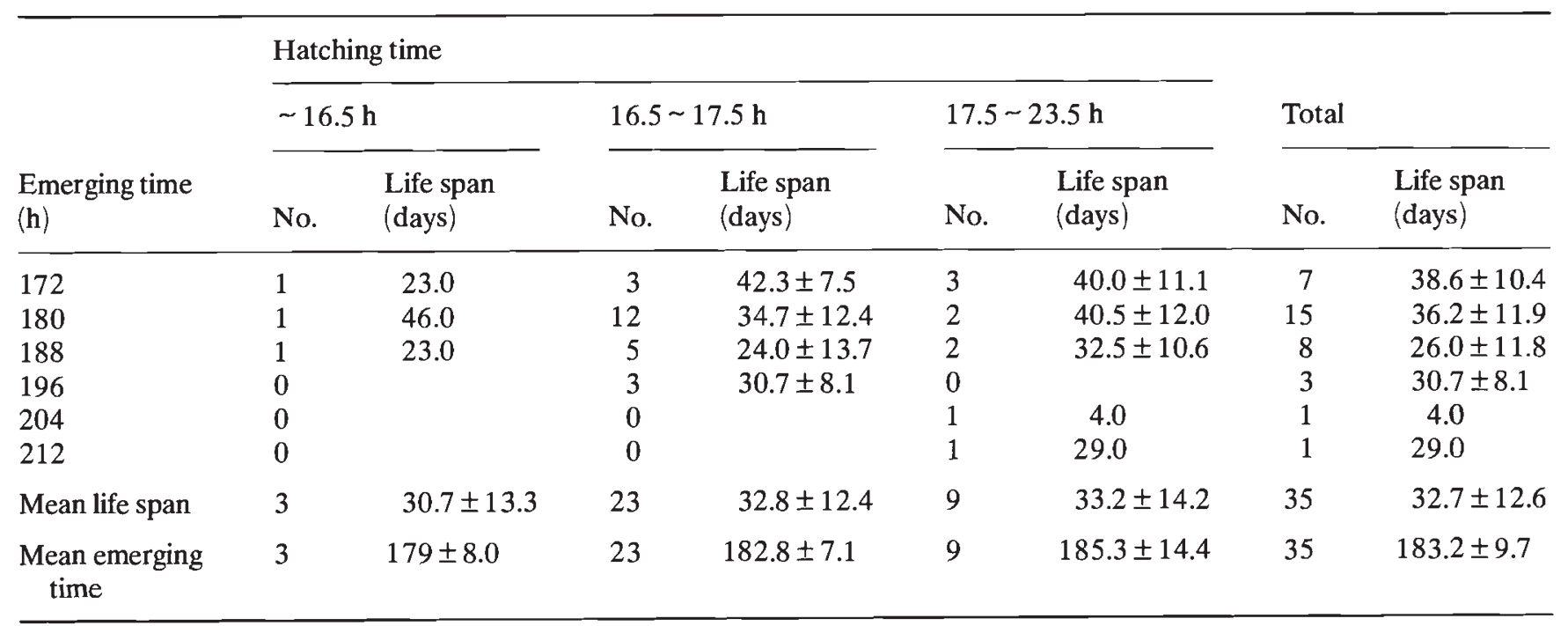

Values are mean \pm s.d.

Table 4 The mean adult life span and emerging time for each hatching time group (females)

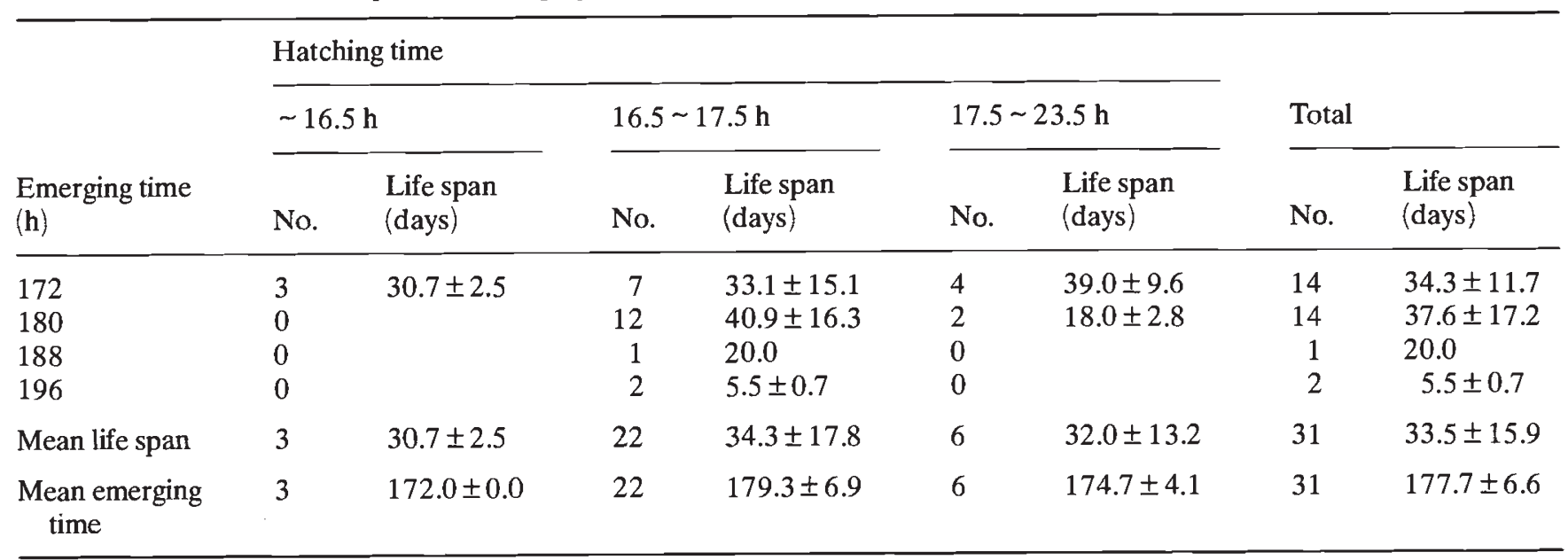

Values are mean \pm s.d. 
known to exist and studied for more than 30 years by Durrant (1955) and Wattiaux (1962). They have warned that procedures used to sample emerging adult flies are important for genetic studies of quantitative traits. The life span is a quantitative trait whose relation with the rate of development in $D$. melanogaster was investigated by Burcombe \& Hollingsworth $(1970)$ and Lints \& Lints (1971). They reported that a longer developmental period was associated with a longer adult life span. In order to compare the adult life span of individuals with different rates of development, Burcombe \& Hollingsworth altered the breeding temperature, and Lints \& Lints changed larval density at a constant temperature. They postulated that changes in environmental factors caused functional changes in physical molecules (Burcombe \& Hollingsworth, 1970) or delay the translation of vital programmes (Lints \& Lints, 1971) resulting in retardation from larval development to senile death.

Recently, Economos \& Lints (1984a,b) and Lints (1989) produced a variety of flies with various rates of development and investigated their adult life span. They reported that this was longest in individuals with an optimum rate of development, and shorter in those with a slower or faster rate. They did not discuss the possible mechanism of the relation.

Two questions should be asked: (1) is the rate of development related to the adult life span and if so, (2) what is the cause and what is the result?

In this study, the mean life span of groups of flies emerging at different times differed significantly. Earlier emergers belonged to the long-lived group and possessed $\operatorname{Jm} A 2$, an autosomal longevity gene which gives longer life. As the generation that segregated short-lived $(A 1 A 1)$ and long-lived $(A 1 A 2)$ genotypes was bred in a constant environment, then the differences in the rate of development can be attributed to genetic differences. We can conclude therefore, that the Jm longevity gene system contributes to determining the rate of development, $\operatorname{Jm} A 2$, the long-life allele, results in faster emergence than $\mathrm{Jm} A 1$, the short-life allele.

Some unexpected results were noted in the Emerging speed/Life span Experiment. A significant correlation was not observed between developmental speed and adult life span in one of six culture bottles; a few long-lived individuals appeared to slow rates of development groups (time groups 8 and 9 in Table 1). Their genotype was assumed to be $A 1 A 2$ as it was repeatedly observed that no individual could have a long-life who lacked $\mathrm{Jm} A 2$ gene (Yonemura et al., 1989; Yonemura et al., 1990). These might therefore reflect some unusual events, e.g. mutation or deletion of other genes, which are important to development.
The present study found no correlation between hatching time and adult life span. It seems that the rate of development in the embryonic period (pre-hatching stage) is not influenced by the Jm longevity genes. As emerging time correlates significantly with adult life span, Jm genes are presumed to have some effect on the rate of development in a period between hatching and emerging. These conclusions are supported by the lack of a correlation between hatching time and emerging time in the present study.

Although we have stated that larvae in the same bottle share an identical environment, it is possible that flies which hatch early obtain food in a different (better) condition than those which hatch late. If larval density is too high, late hatchers (i.e. short-lived) may well suffer a disadvantage and may not survive, or may have decreased reproduction. This might explain the experimental results of Lints \& Lints (1971) who found that breeding larvae in high density delayed development and increased the adult life span, or those of Luckinbill \& Clare (1985) who succeeded in selecting long-lived individuals by high larval density combined with late reproduction in the adult period.

The present experiment was performed under constant conditions $\left(27^{\circ} \mathrm{C}\right.$, food with 8 per cent dry yeast and 5 per cent sugar). It is possible that environmental changes may reveal different results and new findings. Now that longevity genes have been found to exert influences on the rate of development, further investigations are necessary on morphological (e.g. bristle numbers), physiological and biochemical traits.

Participation in the rate of development, as demonstrated here, may be the earliest function of the $\mathrm{Jm}$ longevity genes. As this activity occurs in the preimaginal period, then differentiation of the life span or longevity potential in $D$. melanogaster may also take place during this period. If this assumption is correct, investigation of the precise period of the expression of longevity gene function, and the mechanism of controlling the rate of development would be a most promising approach towards clarification of the mechanism of genetic determination of the life span.

\section{Acknowledgements}

This study was supported by the Smoking Research Foundation(Japan).

\section{References}

ALPATOV, W. W. AND PEARL, R. 1929. Experimental studies on the duration of life. XII. Influence of temperature during the larval period and adult life on the duration of the life of imago of Drosophila melanogaster. Am. Nat., 63, 37-67. 
BURCOMBE, J. V. AND HOLLINGSWORTH, M. J. 1970. The relationship between developmental temperature and longevity in Drosophila. Gerontologia, 16, 172-181.

COHET, v. 1975. Epigenetic influences on the lifespan of the Drosophila: existence of an optimal growth temperature for adult longevity. Exp. Gerontol., 10, 181-184.

DURRANT, A. 1955. Effect of time of embryo formation on quantitative characters in Drosophila. Nature, 175, 560-561.

ECONOMOS, A. C. AND LinTS, F. A. 1984a. Growth rate and life span in Drosophila. I. Methods and mechanisms of variation of growth rate. Mech. Age Dev., 27, 1-13.

ECONOMOS, A. C. AND LinTs, F. A. 1984b. Growth rate and life span in Drosophila. II. A biphasic relationship between growthrate and life span. Mech. Age Dev., 27, 143-151.

LINTS, F. A. 1989. The rate of living theory revisited. Gerotology, 35, 36-57.

LINTS, F. A. AND LINTS, C. v. 1971. Relationship between growth and ageing in Drosophila melanogaster. Nature, 229, $86-88$.
LUCKINBILL, L. S. AND CLARE, J. 1985. Selection for life span in Drosophila melanogaster. Heredity, 55, 9-18.

LUCKINBILL, L. S., GRAVE, J. L., REED, A. H. AND KOESTAWANG, S. 1987. Localizing genes that defer senescence in Drosophila melanogaster. Heredity, 60, 367-374.

SOLIMAN, M. H. AND LINTS, F. A. 1982. Influence of preimaginal constant and alternating temperatures on growth rate and longevity of adults of five genotypes in Tribolium castaneum. Mech. Age Dev., 18, 19-31.

WATTIAUX, J. M. 1962. Variation of bristle number in relation to speed of development in Drosophila melanogaster. Nature, 194, 706-707.

YONEMURA, I., ABE, M., ISHIDATE, R., ISHIYAMA, T., MOTOYAMA, T., HASEKURA, H. AND BOETTCHER, B. 1990. Influence of temperature on the inheritance of adult life span in Drosophila melanogaster. Hereditas, 112, 117-127.

YONEMURA, I., MOTOYAMA, T. AND HASEKURA, H. 1989. Mode of inheritance of major genes controlling life span differences between two inbred strains of Drosophila melanogaster. Hereditas, 111, 207-214. 